\title{
Self-Regulation, Ego Depletion, and Motivation
}

\author{
Roy F. Baumeister ${ }^{1}$ and Kathleen D. Vohs ${ }^{2 *}$ \\ ${ }^{1}$ Florida State University \\ ${ }^{2}$ University of Minnesota
}

\section{Abstract}

Motivation is underappreciated in self-regulation theories (as is true in social personality psychology at large). This paper reviews the role of motivation in the context of the strength, or limited-resource, model of self-control in several domains. Sacrificing one desire in order to pursue another is more difficult when the incipient response is strongly motivated, a notion that highlights the struggle between urges and restraints. A reduction in ego resources can be temporarily overcome by strong motivation - nevertheless, ego depletion is not solely a loss of motivation: Recent experiments indicate that regulatory resources are rooted in physical energy stores. Motivational conflicts, especially the clash between selfish motives and behaviors that promote social acceptance, set the stage for the necessity of self-regulation and the circumstances in which ego depletion is most likely.

Self-regulation is the self's capacity for altering its behaviors. It greatly increases the flexibility and adaptability of human behavior, enabling people to adjust their actions to a remarkably broad range of social and situational demands. It is an important basis for the popular conception of free will and for socially desirable behavior. It provides benefits to the individual and to society, and indeed good self-control seems to contribute to a great many desirable outcomes, including task performance, school and work success, popularity, mental health and adjustment, and good interpersonal relationships (Baumeister, Heatherton, \& Tice, 1994; Duckworth \& Seligman, 2005; Mischel, Shoda, \& Peake, 1988; Shoda, Mischel, \& Peake, 1990; Tangney, Baumeister, \& Boone, 2004; Wolfe \& Johnson, 1995).

Motivation is underappreciated in psychology generally, no doubt partly because the cognitive revolution of recent decades has induced the majority of researchers to think in cognitive rather than motivational terms. Motivation's role in self-regulation has been similarly underestimated. This article seeks to remedy that deficiency. It will examine the multiple ways that motivation is relevant to self-regulation. The relevance to self-regulation spans a wide range, from the confluence of motivation and self-regulation (as in the motivation to engage in self-regulation) to their conflict (as when self-regulation is used to restrain motivation). 
In this article we examine research on self-regulation and the related notion of willpower in relation to motivation. Self-regulation is often employed to restrain motivations, but the motivation to self-regulate is often crucial to the success of engaging in self-regulation.

\section{Definitions}

The term motivation can be used in different ways, but in essence it refers to any sort of general drive or inclination to do something. Cognition, which has dominated psychological theory in recent decades, is ultimately just a tool in service of motivation. That is, the reason evolution selected in favor of increased powers of thought is that those increased powers enable people to get what they want and need. If people did not have wants and needs, there would be little or no reason to think. Human agency or the self's executive function, which includes active initiative, choice, and selfregulation, is thus probably an adaptation to facilitate motivated behavior.

Urges or impulses represent an inclination to respond a certain way in a particular situation on a particular occasion. These terms suggest that an energy or force underlies these types of leanings, which sometimes people seek to change. Urges and impulses are specific manifestations of general motivations.

Self-regulation refers to the capacity of organisms (here, human beings) to override and alter their responses. It is the process by which people attempt to constrain unwanted urges in order to gain control of the incipient response. Regulation means change, especially change to bring behavior (or other states) into line with some standard such as an ideal or goal. Changing one's behavior so as to follow rules, match ideals, or pursue goals is thus a (very useful) form of self-regulation. To change a response does not necessarily mean to override it, although self-restraint is a common form of selfregulation (Polivy, 1998), but so is the amplification or prolonging of a response. Still, the most common form of regulation is to override and stifle.

Ego depletion refers to a state in which the self does not have all the resources it has normally. We shall suggest that self's executive function, which includes self-regulation as well as effortful choice and active initiative, depends on a limited resource that is consumed during such activities. Ego depletion renders the self temporarily less able and less willing to function normally or optimally. The motivational aspect of ego depletion is a particular focus of this article.

\section{Ingredients of Self-Regulation}

Previous analyses of self-regulation (e.g., Baumeister et al., 1994) emphasized three main ingredients of the self-regulation process, but we are now convinced that a fourth needs to be included. The fourth ingredient is 
motivation. Next we detail the four component parts, in order to appreciate the proper role of motivation.

The first ingredient is standards. As the definition indicated, regulation means change so as to bring into line with some standard, and hence effective self-regulation requires a clear and well-defined standard. Ambiguous, uncertain, inconsistent, or conflicting standards make self-regulation difficult. Research such as by Higgins (1987) has emphasized how different standards can alter emotional reactions and behavioral processes.

Second, self-regulation requires monitoring. It is difficult if not impossible to regulate a behavior without keeping track of it. Self-regulation theory has been hugely influenced by the seminal work of Carver and Scheier (1981, 1982, 1998). One major aspect of their contribution was to adapt feedback-loop theory to human self-regulation. The person performs a test by comparing the self (or the relevant aspect of self) to the standard. If the self falls short, then self-regulation requires initiating some operation to change the self in order to bring it up to what it should be. Further tests evaluate progress toward meeting the goal and eventually confirm that the self has now been brought into line with the standard, whereupon no further operations are required, and so the operations can cease.

The third ingredient is self-regulatory strength, colloquially known as willpower. Operations aimed at changing the self are difficult and therefore require some power. Regulating the self appears to depend on a limited resource that operates like a strength or energy and becomes temporarily depleted afterward (e.g., Baumeister, Bratslavsky, Muraven, \& Tice, 1998; Muraven \& Baumeister, 2000; Vohs \& Heatherton, 2000), thus creating the state of ego depletion. Recent work has indicated that blood glucose, which is the brain's principal source of fuel, is an important component of this resource: Acts of self-control consume substantial quantities of glucose, resulting in lower levels of it in the bloodstream (Gailliot et al., 2007).

The fourth ingredient is motivation - specifically, motivation to achieve the goal or meet the standard, which in practice amounts to motivation to regulate the self. Even if the standards are clear, monitoring is fully effective, and the person's resources are abundant, he or she may still fail to self-regulate due to not caring about reaching the goal.

Thus, the proper way to understand the role of motivation in selfregulation is as one of four ingredients. As the term ingredients implies, some of each is necessary for effective self-regulation. However, it is possible that the four can compensate or substitute for each other to some degree. If motivation is high, such as if the person really and strongly wants to measure up to some standard, this may compensate for a somewhat lower than usual level of willpower or a greater difficulty of monitoring. For example, alcohol impairs self-regulation, in large part by impairing the monitoring of one's behavior (e.g., Hull, 1981), and so an intoxicated person may be prone to say or do the wrong thing. But if motivation is high - perhaps the person's boss or father-in-law unexpectedly enters the scene - then the person may 
still manage to speak carefully and properly, despite the greater difficulty. Such compensation will only work up to a point, but it does suggest one potentially important role of motivation.

Motivation may be especially effective at substituting for willpower. Even if willpower (i.e., self-regulatory strength) has been depleted by prior acts, the person may be able to self-regulate effectively if motivation is high, as we shall see. In contrast, motivation may not be enough to substitute for the lack of a clear standard. That combination would mean someone wanting to self-regulate but not knowing what were desirable responses to enact. As for monitoring, we have suggested that motivation may sometimes be able to offset impaired capacity to self-monitor, but this effect may be limited (which is why people should avoid being drunk at job interviews). To be sure, there may be degrees of depleted willpower beyond which no amount of motivation can compensate, although this notion remains to be shown empirically.

Motivation is subject to change in response to a variety of circumstances. Up until now, self-regulation theory has paid little attention to the issues of whether and how strongly people are motivated to regulate themselves. But it seems plausible that, like other motivations, the motivation to self-regulate would fluctuate according to anticipated satisfactions, expected utility, efficacy expectations, and other factors.

\section{Self-Regulation and Motivation: Friends or Enemies?}

Why do animals (and especially human animals) have self-regulation in the first place? Without motivation, there would be little need for it. Self-regulation is first and foremost useful to prevent some impulses, which are based in motivations, from being enacted in behaviors that would be costly to the individual, even if they promise short-term benefits in terms of satisfying current needs and wants. Thus, some basic uses of self-regulation are inimical to motivation.

While conducting an early review of the self-regulation literature (Baumeister et al., 1994), one of us noticed the pattern that the most common function of self-regulation is to stop impulses from producing behavior (also Polivy, 1998). Most, although not all, self-regulation can be understood as self-stopping: The dieter refrains from eating, the recovering addict refrains from drinking, smoking, or drug use, the anger-manager holds his or her tongue and refrains from doing something that will be regretted later, and so forth. Essentially, the majority of self-regulation functions to thwart and prevent motivated behavior.

Then again, we must ask, why do people want to restrain their motivations? After all, motivations exist for beneficial - even adaptive reasons. The motivation to find and eat food is based on the body's need for nutrients in order to survive. Sexual motivation reflects the importance of engaging in reproductive activities, and animals without sexual motivation 
may well fail to pass on their genes. Motivations to seek affiliation, intimacy, and power reflect the fact that social and cultural life are the principal biological strategies of human beings, insofar as success at interpersonal and group life are the main ways that people achieve survival and reproduction.

Why, then, would nature give humans the capability to restrain their motivations, when it has instilled those same motivations via natural selection? Motivational conflict is the crucial answer and provides the essential context for understanding self-regulation. When a person wants something and wants it without question or conflict, such as when one's canoe is sinking and one needs to swim to shore so as not to drown, there is no motivational conflict and hence no need for self-regulation. But when motivations clash, self-regulation becomes needed and may be the key to success. For example, humans evolved to find, eat, and store as many calories as possible, but the success of culture has changed the environment from one of chronic famine to one of chronic abundance. Modern Westerners who eat everything they want to eat eventually grow fat, which is detrimental to their health and their social standing. A motivational conflict therefore ensues: The desire to eat delicious food conflicts with the desire to be healthy (and fashionably slender!). Self-regulation is needed in order to resist the natural desire to eat good-tasting and easily available food.

Perhaps the most important motivational conflict arises from the clashing demands of nature and culture. Baumeister (2005) has proposed to view nature and culture as operating largely together, insofar as evolution selected humans for traits that made them competent to create and function in the complex social systems that became culture. However, short-term self-interest remains a chronic nexus of conflict. Selfishness is natural: Brains and a great many psychological traits evolved, after all, to enable the organisms that housed them to survive and reproduce successfully. Animals that failed to do what was best for themselves and their immediate kin were most likely supplanted by rivals who did. Culture, meanwhile, is a group system, and sometimes what is best for the group is not what is best for the individual. Waiting one's turn, respecting the property of others, paying taxes, and risking one's life in battle are examples of behaviors that are beneficial for the group but that come at cost to individuals. None of those behaviors comes naturally, and in fact it may be natural to have impulses to do the opposite. Self-regulation is therefore helpful and perhaps vital for culture to succeed.

The crucial motivational conflict in these clashes between natural impulses and cultural demands lies in the desire for social acceptance. The need to belong is one of the most basic and pervasive human drives. Baumeister and Leary (1995) reviewed considerable evidence that this need is strong among people generally and that it underlies a broad range of cognitive, emotional, and behavioral responses. In a similar vein, self-determination theorists (Deci \& Ryan, 2000) have concluded that the desire for social connection is one 
of the three most basic and powerful human motives. That motive is ultimately what comes into conflict with selfish impulses (Heatherton \& Vohs, 1998). In the moment, perhaps, selfish people may be more likely than others to get what they want, but others quickly begin to avoid them (for good reason!). Hence, people learn that restraining selfishness is helpful and even necessary in order to gain social acceptance (Heatherton \& Vohs, 1998).

One might ask why people care so much about social acceptance. In our view, this reflects basic biological strategies of human life. Both survival and reproduction are far easier to achieve via social acceptance than in social isolation (see Bowlby, 1969). More broadly, culture is a biological strategy for humans (Baumeister, 2005), and humans get what they need for survival more often from their social group than directly from nature, unlike most other social animals. Social acceptance is not a whim or luxury but rather a central drive tied to the most basic needs for survival and reproduction. Indeed, this has been one of the critiques of Maslow's much-beloved theory of the hierarchy of needs (Wahba \& Bridwell, 1976). According to Maslow's hierarchy, the drive for belongingness emerges only after the more basic needs for food, shelter, and safety and security are satisfied. Only when the belly is full and the predators and other dangers thwarted does the human psyche begin to care about forming and affirming social bonds. Observations suggest, however, that hunger and danger often stimulate the desire to affiliate with others, or at least to seek out trusted relationship partners (Schachter, 1959; Taylor et al., 2000). Perhaps most notably, people will risk life and limb for the sake of belongingness (e.g., Leary, Tchividjian, \& Kraxberger, 1994).

To return to our theme of the roots of self-regulation, we conclude that much of self-regulation is used for the purpose of restraining selfish motivations in order to serve the goal of being accepted by others (Heatherton \& Vohs, 1998). In an important sense, this is a matter of pursuing enlightened self-interest over immediate or myopic self-interest.

\section{Hard and Easy Sacrifices}

Thus far we have suggested that self-regulation typically occurs to resolve motivational conflict. One implication is that the ease and presumably the success of the self-regulation task will depend in substantial part on the relative strengths of the competing motivations. Put another way, effective self-regulation often involves sacrificing something one wants in order to achieve something else that one wants even more. Sacrificing the first outcome will become more difficult in proportion to how much one wants it. Conversely, the more one wants to achieve the second (more enlightened or long-term) goal, the easier it may be to make sacrifices for it.

Sexuality research has provided evidence toward the argument that controlling an urge is more difficult as the urge becomes stronger. The 
stronger sex drive in men makes it more difficult to refrain from sexual behavior (Baumeister, Catanese, \& Vohs, 2001). For example, both priests and nuns take a vow to abstain forever from all sexual activity. Nonetheless, not only do priests masturbate more than nuns (Sipe, 1995), more priests than nuns engage in sexual activity after their commitment to celibacy (Murphy, 1992). A series of experiments in the laboratory on sexuality and self-regulatory capacity suggested that men's sexual responsiveness may also be more affected by lowered self-control than women (Gailliot \& Baumeister, 2007). Ego depletion released more sexual behavior in men than women.

Although we do not know of research directly testing the hypothesis that self-regulatory success depends on the relative strength of the competing motivations, some findings can be interpreted in that way. Research on relationships provides some evidence. Studies by Vohs, Finkenauer, and Baumeister (2007) established that self-regulation benefits relationships in part by directing attention away from alternative partners who might tempt one away. Specifically, they showed that when people's self-regulatory resources are depleted, they spend more time attending to photos of attractive members of the opposite sex. Miller (1997) showed that attending longer to such photos predicts a higher rate of relationship dissolution in the months after the experiment. One way of interpreting Miller's findings is that people who are less motivated to sustain their current relationships are less willing to make the self-regulatory effort and sacrifice to avoid temptation. In the laboratory, they looked at pictures, and presumably outside the laboratory they would flirt and respond and in other ways that may allow themselves to become attracted to alternative partners that eventually could replace their primary relationship.

We noted that motivational strength can change over time, and such changes seem especially likely in relationships. At the height of passionate love, partners might be willing to sacrifice their lives for each other, whereas several years down the road they might not even bother to bring the partner the remote control or to interrupt their fun to phone home.

The analysis thus far has suggested that the incentive value of the primary relationship (and therefore the strength of the motivation to sustain it) may affect the willingness to make sacrifices for it. The strength of the competing motivation, such as to embrace alternative sexual satisfaction, may also affect the self-regulatory outcome. Even if both partners have exactly the same degree of commitment to the relationship and hence exactly the same motivation to do whatever is best for it, one may be more likely than the other to engage in sex with someone else, if one person has a much stronger desire for such sexual activity than does the other. In heterosexual romantic relationships, generally, the man is likely to be that person. Evidence points rather consistently to the conclusion that men desire sex more than women (Baumeister et al., 2001), and the desire for multiple partners may be much stronger and more natural in men than women (Haselton \& Buss, 2000). As a result, nearly all surveys indicate that husbands are more likely than wives 
to engage in extramarital sex (Laumann, Gagnon, Michael, \& Michaels, 1994; Spanier \& Margolis, 1983). The gender difference in extramarital couplings is likely due more to differences in urges than to differences in commitment (Baumeister et al., 2001).

\section{Hard and Easy Choices}

Earlier we suggested that ego depletion can stem from effortful choice as well as from self-regulation. The first study to extend ego depletion to choice was by Baumeister et al. (1998). It used a dissonance paradigm, which by definition invokes motivational conflict: Dissonance theory is essentially based on consistency motivation, and dissonance arises from inconsistency (Festinger, 1957). Participants were induced to agree to make a speech contrary to their beliefs (specifically, favoring a substantial increase in their tuition for the upcoming year). Making this decision depleted their resources, as indicated by quitting faster on a subsequent task. Perhaps surprisingly, participants in another condition also showed the same pattern of depleted resources and low persistence after making a decision to give a speech consistent with their opinion. That situation clearly did not have the same level of motivational conflict. Still, it seems plausible that many students do not want to make recorded speeches at all, and so consenting to speak could have presented a motivational conflict for them. (Presumably they also did not want to refuse the experimenter's request.)

Early research on stress has provided some support for the view that difficult decisions instigate costly inner processes. Weiss (1971) put rats in a situation in which they had to make a bar press response to avoid shock but successful avoidance was signaled by an aversive blast of loud noise. In effect, the rat had to choose the lesser of two evils (shock versus noise). These rats developed ulcers comparable to those of the 'helpless' rats who were exposed to inescapable shock, even though in general avoidance options produced substantial reductions in stress. Choosing the lesser of two evils is thus apparently a stressful dilemma, even for rats.

Recent studies provide further support for the conclusion that depletion comes from the degree of struggle. Participants in studies by Vohs, Baumeister, et al. (2007) had to make a series of choices among consumer products or among options for their psychology course. They were free to choose whatever they liked and yet they exhibited ample behavioral signs of ego depletion afterward, including reduced persistence on tasks, poorer performance at cold pressor, and reduced consumption of a healthy but bad-tasting beverage.

To investigate the role of inner effort, Vohs, Finkenauer, et al. (2007) conducted two further studies. In one, participants executed preordained choices without thinking about them, or deliberated between options without choosing, or did both deliberating and choosing. The last was the most depleting, whereas executing choices without deliberating was the 
least depleting. Deliberating without choosing still depleted some resources, but not as much as deliberating and choosing. The choice process thus consumes some resources above and beyond the process of merely thinking about the options.

In a final study, participants performed a bridal registry task (making many choices of gifts they would like to receive at their wedding) for 4 minutes or 12 minutes or, in the control condition, made no choices. Their liking for the task was measured, and sure enough some participants found the task fun whereas others detested it. Afterward, depletion was measured by passivity: Participants were seated at a computer and told to wait for instructions, and the computer simply stayed on blue screen, so eventually the participant had to find the experimenter and explain that the computer was apparently malfunctioning. Liking for the task interacted with duration of task. When the choosing task was brief (4 minutes), those who disliked the task were depleted by making the choices, whereas those who enjoyed the task showed little or no sign of depletion. However, when the choosing task extended to 12 minutes, thus requiring more effort even for those who enjoyed it, all participants showed signs of depletion regardless of whether they had liked or disliked the bridal registry task. This study showed that when choosing is fun, it takes longer for the depleting effects to emerge than when choosing is aversive. Hence, overall making a series of choices is still somewhat depleting.

In summary, the data suggest that there are debilitating effects of choosing on later self-regulation. After making many choices, the chooser is less able to engage in good self-control, suggesting that making choices exhausts the self over time.

\section{Motivation After Depletion}

Thus far we have focused on how resources get depleted by coping with motivation and, in particular, managing motivational conflict. Now we turn to the other side of the coin, namely examining the role of motivation when resources are already depleted. This will involve a shift from treating motivation as the enemy of self-regulation and instead focusing on how the two can work together.

We proposed earlier that motivation sometimes substitute for deficient ability. This was tested directly by Muraven and Slessareva (2003), who showed that depleted participants can effectively self-regulate if they are offered an incentive to do so. In one study, participants whose resources had been depleted by a prior self-regulatory task showed the standard drop in subsequent self-regulation, insofar as they consumed relatively little of a healthy but bad-tasting beverage. However, when they were offered a cash incentive to drink more, they consumed a substantial amount - if anything more than non-depleted participants, rather than less. Thus, the motivational incentive completely erased the effect of ego depletion. 
It is tempting to interpret Muraven and Slessareva's (2003) findings as indicating that ego depletion is essentially a state of motivation, but we think that would be a misinterpretation. The analogy to physical tiredness is illuminating. Physically tired people generally perform worse than others at strenuous tasks, but if the incentive is high enough, they can perform well despite their tiredness. The fact that high motivation can overcome the effect of tiredness does not mean that tiredness is nothing more than a lack of motivation. Indeed, researchers in various laboratories have begun to explore various antidotes to ego depletion. Webb and Sheeran (2002) showed that forming implementation intentions can enable people to function well despite ego depletion, but this does not mean that ego depletion is essentially a lack of implementation intentions. Tice, Baumeister, Shmueli, and Muraven (forthcoming) found that watching a humorous video can cause people to perform well after depletion, but this does not mean that ego depletion is essentially a lack of something to laugh about. Cognitive factors too can boost motivation and overcome some depletion effects. Getting people to believe that they can do it seems to help them keep up with a controlled response, such as squeezing a handgrip (Martijn, Tenbült, Merckelbach, Dreezens, \& deVries, 2002), but again that does not mean that ego depletion is mainly a lack of self-confidence. In summary, the most plausible conclusion is that motivation (like cognition and perhaps emotion) can compensate for the reduced ability to self-regulate that ordinarily marks the depleted state.

One important version of motivational accounts of ego depletion is based on the notion that participants are only willing to exert or endure a certain amount for an experiment. In this view, participants use up their good will during the first (depleting) task, which is typically less than pleasant, and therefore they do not want to exert themselves on the second task. There are by now ample findings that cannot be explained on this basis (see Baumeister, Gailliot, DeWall, \& Oaten, 2006, forthcoming), but it is plausible that some of them could fit. To test this, Gailliot et al. (2007) conducted a study in which the depletion manipulation was followed by giving some participants an ice cream treat, which would presumably restore good will. It succeeded in counteracting the effects of depletion, insofar as participants subsequently persisted longer on the next task. However, and unfortunately for the good will motivational account, participants in another condition received a rather unappetizing treat made with tasteless dairy rather than ice cream, and they too performed better on the second task. Thus, the enjoyment was apparently irrelevant. These findings led to the hypothesis that ego depletion may involve a temporary depletion of the body's caloric energy supplies. In a series of studies by Gailliot et al. (2007), depletion was counteracted by snacks that contained glucose but not by equally tasty snacks that provided no glucose.

Thus, it appears that the discussion of self-regulation and ego depletion in terms of energy processes (even the term 'willpower') go beyond mere metaphor (see Gailliot \& Baumeister, forthcoming). Effective self-regulation 
seems to involve utilizing the glucose in the bloodstream to achieve what is a psychologically difficult and biologically costly task, such as stifling one's behavioral impulses or making difficult choices. When glucose - the primary source of fuel for all brain processes - has been depleted, the person is temporarily less able to function at optimal levels. How can motivation (or cognition or emotion) counteract this?

The power of motivation to overcome depletion may lie in the fact that the depleted state does not reflect a complete exhaustion of resources but merely a temporary deficit. Like a tired athlete who starts conserving energy long before he or she is completely exhausted, the self-regulator may begin to cut back on effortful, biologically expensive exertions long before the capacity is fully depleted. Ego depletion effects thus indicate conservation of a partly depleted resource, rather than full incapacity because the resource is completely gone. Hence, motivations and incentives can inspire the person to expend some of the remaining resource even when it is depleted. That, presumably, is the point of conservation: to save some of the resources in case of pressing need or exceptional opportunity.

The conservation hypothesis was supported in a series of studies by Muraven, Shmueli, and Burkley (2006). After an initial depleting task, participants performed a second task, with the twist that some of them were told that a subsequent (third) task would require self-control strength. The depletion effects on the second task were most pronounced among participants who expected the third task, indicating that they were conserving some of their resources for the third task. Furthermore, performance on the third task was found to depend on how the person had done on the second - inversely. The less the person had self-regulated on the second task, the better he or she did on the third. Conservation thus does seem to work. Had the second task been more important from a motivational perspective, the person would presumably have self-regulated more on it, thereby expending even more of the depleted resource, with the result that there would have been much less remaining for the third task.

The conservation findings shed light on why motivation can counteract depletion. If the tank were truly and thoroughly empty, it is unlikely that increasing incentives would counteract depletion. But if we understand depletion effects as a natural and adaptive pattern of conserving what remains of a valuable and limited resource, then providing incentives to motivate further self-regulation could persuade the person to spend more of that resource.

\section{Conclusion}

Motivation is fundamental to life, and indeed most likely the self as agent evolved to facilitate the goal pursuits associated with crucial motivations. Yet in order to manage conflicts between inner motivations and between external constraints and inner motives, self-regulation is a vital function of the agentic 
self (Baumeister, 1998). Recent self-regulatory theories have probably not given sufficient attention to the role played by motivation in enabling self-regulation to be successful - even when self-regulation is employed to restrain motivation.

\section{Short Biography}

Roy F. Baumeister is the Francis Eppes Eminent Scholar and Professor of Psychology at Florida State University, where he also serves as head of the social psychology graduate program. He received his $\mathrm{PhD}$ in social psychology in 1978 from Princeton University, and his research and teaching have also taken him to the University of California at Berkeley, Case Western Reserve University, the University of Texas at Austin, the Max-Planck-Institute for Psychological Research in Munich (Germany), the University of Virginia, and the Center for Advanced Study in the Behavioral Sciences (at Stanford). He has authored nearly 350 publications, and the principal outlets for his best work include the Journal of Personality and Social Psychology as well as Psychological Bulletin. He has written or edited 20 books, including Evil: Inside Human Violence and Cruelty (Freeman, 1997), The Cultural Animal: Human Nature, Meaning, and Social Life (Oxford, 2005), and the Handbook of Self-Regulation (with K. Vohs; Guilford, 2004), as well as the new social psychology textbook, Social Psychology and Human Nature (with B. Bushman; Wadsworth, 2008). In addition to the textbook, his recent educational activities include teaching courses in social psychology and taking lessons in kitesurfing. His research is currently funded by the Templeton Foundation and the National Institute of Mental Health. He studies a broad range of topics, including self-control, the need to belong, sexuality, aggression, social rejection, power, self-esteem, consciousness, free will, gender, and culture.

Kathleen D. Vohs is Assistant Professor in the Department of Marketing, Carlson School of Management, University of Minnesota. Vohs received her PhD in Psychological and Brain Sciences from Dartmouth College in 2000 after which she conducted research at the University of Utah and Case Western Reserve University. In 2003, she joined the Marketing Division at the University of British Columbia, where she was awarded the Canada Research Chair in Marketing Science and Consumer Psychology. In 2007, Vohs was named a McKnight Land-Grant Professor at the University of Minnesota. Vohs has contributed over 80 professional publications, including coediting three books and one Encyclopedia. Her theories highlight the role of the self and her research has been extended to the domains of chronic dieting, bulimic symptoms, sexuality, impulsive spending, and compulsive spending. Her work has been funded by the National Institutes of Health, the Social Sciences and Humanities Council of Canada, and the American Cancer Society. When not working, Vohs likes wine tasting and attending yoga classes (not simultaneously). 


\section{Endnote}

^ Correspondence address: Department of Marketing, Carlson School of Management, University of Minnesota, 3-150, 321 19th Ave. South, Minneapolis, MN 55455, USA. Email: kvohs@carlsonschool.umn.edu.

\section{Bibliography}

Baumeister, R. F. (1998). The self. In D. T. Gilbert, S. T. Fiske, \& G. Lindzey (Eds.), Handbook of Social Psychology (4th edn, pp. 680-740). New York: McGraw-Hill.

Baumeister, R. F. (2005). The Cultural Animal: Human Nature, Meaning, and Social Life. New York: Oxford University Press.

Baumeister, R. F., Bratslavsky, E., Muraven, M., \& Tice, D. M. (1998). Ego depletion: Is the active self a limited resource? Journal of Personality and Social Psychology, 74, 1252-1265.

Baumeister, R. F., Catanese, K. R., \& Vohs, K. D. (2001). Is there a gender difference in strength of sex drive? Theoretical views, conceptual distinctions, and a review of relevant evidence. Personality and Social Psychology Review, 5, 242-273.

Baumeister, R. F., Gailliot, M., DeWall, C. N., \& Oaten, M. (2006). Self-regulation and personality: How interventions increase regulatory success, and how depletion moderates the effects of traits on behavior. Journal of Personality, 74, 1773-1801.

Baumeister, R. F., Heatherton, T. F., \& Tice, D. M. (1994). Losing Control: How and Why People Fail at Self-Regulation. San Diego, CA: Academic Press.

Baumeister, R. F., \& Leary, M. R. (1995). The need to belong: Desire for interpersonal attachments as a fundamental human motivation. Psychological Bulletin, 117, 497-529.

Bowlby, J. (1969). Attachment and Loss: Vol. 1. Attachment. New York: Basic Books.

Carver, C. S., \& Scheier, M. F. (1981). Attention and Self-Regulation: A Control Theory Approach to Human Behavior. New York: Springer-Verlag.

Carver, C. S., \& Scheier, M. F. (1982). Control theory: A useful conceptual framework for personality-social, clinical and health psychology. Psychological Bulletin, 92, 111-135.

Carver, C. S., \& Scheier, M. F. (1998). On the Self-Regulation of Behavior. New York: Cambridge University Press.

Deci, E. L., \& Ryan, R. M. (2000). The 'what' and 'why' of goal pursuits: Human needs and the self-determination of behavior. Psychological Inquiry, 11, 227-268.

Duckworth, A. L., \& Seligman, M. E. P. (2005). Self-discipline outdoes IQ in predicting academic performance of adolescents. Psychological Science, 16, 939-944.

Festinger, L. (1957). A theory of Cognitive Dissonance. Stanford, CA: Stanford University Press.

Gailliot, M. T., \& Baumeister, R. F. (2007). Self-regulation and sexual restraint: Dispositionally and temporarily poor self-regulatory abilities contribute to failure at restraining sexual behavior. Personality and Social Psychology Bulletin, 33, 173-186.

Gailliot, M. T., \& Baumeister, R. F. (forthcoming). The physiology of willpower: Linking blood glucose to self-control. Personality and Social Psychology Review.

Gailliot, M. T., Baumeister, R. F., DeWall, C. N., Maner, J. K., Plant, E. A., Tice, D. M., Brewer, L. E., \& Schmeichel, B. J. (2007). Self-control relies on glucose as a limited energy source: Willpower is more than a metaphor. Journal of Personality and Social Psychology, 92, 325-336.

Haselton, M., \& Buss, D. (2000). Error management theory: A new perspective on biases in cross-sex mind reading. Journal of Personality and Social Psychology, 78, 81-91.

Heatherton, T. F., \& Vohs, K. D. (1998). Why is it so difficult to inhibit behavior? Psychological Inquiry, 9, 212-215.

Higgins, E. T. (1987). Self-discrepancy: A theory relating self and affect. Psychological Review, 94, 319-340.

Hull, J. G. (1981). A self-awareness model of the causes and effects of alcohol consumption. Journal of Abnormal Psychology, 90, 586-600.

Laumann, E. O., Gagnon, J. H., Michael, R. T., \& Michaels, S. (1994). The Social Organization of Sexuality: Sexual Practices in the United States. Chicago, IL: University of Chicago Press. 
Leary, M. R., Tchividjian, L. R., \& Kraxberger, B. E. (1994). Self-presentation can be hazardous to your health: Impression management and health risk. Health Psychology, 13, 461-470.

Martijn, C., Tenbült, P., Merckelbach, H., Dreezens, E., \& de Vries, N. K. (2002). Getting a grip on ourselves: challenging expectancies about loss of energy after self-control. Social Cognition, 20, 441-460.

Miller, R. S. (1997). Inattentive and contented: Relationship commitment and attention to alternatives. Journal of Personality and Social Psychology, 73, 758-766.

Mischel, W., Shoda, Y., \& Peake, P. K. (1988). The nature of adolescent competencies predicted by preschool delay of gratification. Journal of Personality and Social Psychology, 54, 687-696.

Muraven, M., Shmueli, D., \& Burkley, E. (2006). Conserving self-control strength. Journal of Personality and Social Psychology, 91, 524-537.

Muraven, M., \& Slessareva, E. (2003). Mechanism of self-control failure: Motivation and limited resources. Personality and Social Psychology Bulletin, 29, 894-906.

Muraven, M. R., \& Baumeister, R. F. (2000). Self-regulation and depletion of limited resources: Does self-control resemble a muscle? Psychological Bulletin, 126, 247-259.

Murphy, S. (1992). A Delicate Dance: Sexuality, Celibacy, and Relationships among Catholic Clergy and Religious. New York: Crossroad.

Polivy, J. (1998). The effects of behavioral inhibition: Integrating internal cues, cognitive behavior, and affect. Psychological Inquiry, 9, 181-203.

Schachter, S. (1959). The Psychology of Affiliation. Stanford, CA: Stanford University Press.

Shoda, Y., Mischel, W., \& Peake, P. K. (1990). Predicting adolescent cognitive and self-regulatory competencies from preschool delay of gratification: Identifying diagnostic conditions. Developmental Psychology, 26, 978-986.

Sipe, A. W. R. (1995). Sex, Priests, and Power: Anatomy of a Crisis. New York: Brunner/Mazel Publishers.

Spanier, G. P., \& Margolis, R. L. (1983). Marital separation and extramarital sexual behavior. Journal of Sex Research, 19, 23-48.

Tangney, J. P., Baumeister, R. F., \& Boone, A. L. (2004). High self-control predicts good adjustment, less pathology, better grades, and interpersonal success. Journal of Personality, 72, 271-322.

Taylor, S. E., Klein, L. C., Lewis, B. P., Gruenewald, T. L., Gurung, R. A. R., \& Updgraff, J. A. (2000). Biobehavioral responses to stress in females: Tend-and-befriend, not fight-or-flight. Psychological Review, 107, 411-429.

Tice, D. M., Baumeister, R. F., Shmueli, D., \& Muraven, M. (forthcoming). Restoring the self: Positive affect helps improve self-regulation following ego depletion. Journal of Experimental Social Psychology.

Vohs, K. D., Baumeister, R. F., Twenge, J. M., Nelson, N. M., Rawn, C. D., Tice, D. M., \& Schmeichel, B. J. (forthcoming, 2007). Decision Fatigue Exhausts Self-Regulatory Resources. Minneapolis, MN: University of Minnesota. (maniscript under review)

Vohs, K. D., Finkenauer, C., \& Baumeister, R. F. (forthcoming, 2007). Romantic Relationship Functioning Hinges on Self-control Abilities. Minneapolis, MN: University of Minnesota. (manuscript in preparation)

Vohs, K. D., \& Heatherton, T. F. (2000). Self-regulatory failure: A resource-depletion approach. Psychological Science, 11, 249-254.

Wahba, M. A., \& Bridwell, L. G. (1976). Maslow reconsidered: A review of research on the need hierarchy theory. Organizational Behavior and Human Performance, 15, 212-240.

Webb, T. L., \& Sheeran, P. (2002). Can implementation intentions help to overcome ego-depletion? Journal of Experimental Social Psychology, 39, 279-286.

Weiss, J. M. (1971). Effects of punishing the coping response (conflict) on stress pathology in rats. Journal of Comparative and Physiological Psychology, 77, 14-21.

Wolfe, R. N., \& Johnson, S. D. (1995). Personality as a predictor of college performance. Educational and Psychological Measurement, 55, 177-185. 